\title{
Natural Product Compounds in Alpinia officinarum and Ginger are Potent SARS-CoV-2 Papain-like Protease Inhibitors
}

\author{
${ }^{1,4}$ Dibakar Goswami, ${ }^{2,4}$ Mukesh Kumar, ${ }^{1,3,4}$ Sunil K. Ghosh and ${ }^{2,4}$ Amit Das*
}

${ }^{1}$ Bio-Organic Division, ${ }^{2}$ Protein Crystallography Section, Radiation Biology and Health Sciences Division, ${ }^{3}$ Food Technology Division, Bhabha Atomic Research Centre, Trombay, Mumbai-400085. India and ${ }^{4}$ Homi Bhabha National Institute, Anushaktinagar, Mumbai-400094. India.

\begin{abstract}
SARS-CoV-2 or COVID-19 has caused more than 10,00,000 infections and $~ 55,000$ deaths worldwide spanning over 203 countries, and the numbers are exponentially increasing. Due to urgent need of treating the SARS infection, many approved, pre-clinical, anti-viral, anti-malarial and anti-SARS drugs are being administered to patients. SARS-CoV-2 papain-like protease (PLpro) has a protease domain which cleaves the viral polyproteins a/b, necessary for its survival and replication, and is one of the drug target against SARS-CoV-2. 3D structures of SARS-CoV2 PLpro were built by homology modelling. Two models having partially open and closed conformations were used in our study. Virtual screening of natural product compounds was performed. We prepared an in house library of compounds found in rhizomes, Alpinia officinarum, ginger and curcuma, and docked them into the solvent accessible S3-S4 pocket of PLpro. Eight compounds from Alpinia officinarum and ginger bind with high in silico affinity to closed PLpro conformer, and hence are potential SARS-CoV-2 PLpro inhibitors. Our study reveal new lead compounds targeting SARS-CoV-2. Further structure based modifications or extract formulations of these compounds can lead to highly potent inhibitors to treat SARS-CoV2 infections.
\end{abstract}

\section{Keywords}

PLpro, SARS-CoV-2, Molecular docking analysis, Alpinia officinarum, Gingerol, Homology modelling, Natural products.

\author{
Abbreviations \\ PLpro: papain-like protease \\ 3CLpro: 3-chymotrypsin-like protease \\ Nsp: non-structure protein \\ ORF: open reading frame \\ PDB: protein data bank \\ *Corresponding author \\ E-mail : amitdas@barc.gov.in / amitd453@gmail.com
}




\section{Introduction}

Coronaviruses (CoVs) are a large family of viruses that cause diseases ranging from cold to Severe Acute Respiratory Syndrome (SARS). Severe acute respiratory syndrome coronavirus (SARS-CoV) had spread to five continents in 2003 with $10 \%$ fatality [1,2] and Middle East Respiratory Syndrome Coronavirus (MERS-CoV) broke out in the Arabian countries with 35\% fatality [3]. Both SARS-CoV and MERS-CoV are zoonotic viruses with hosts being bat/civet and dromedary camels respectively $[4,5]$. CoVs are enveloped viruses with a positive stranded RNA having large $30 \mathrm{~kb}$ genome, belonging to the Coronaviridae family of the order Nidovirales, and $\beta$ genus. CoVs contain at least four structural proteins: Spike (S) protein, envelope (E) protein, membrane $(\mathrm{M})$ protein, and nucleocapsid $(\mathrm{N})$ protein. Among them, $\mathrm{S}$ proteins promote host attachment and virus-cell membrane fusion during virus infection [6], and also determines the host range [7]. There is no approved therapeutic drug or vaccine for the treatment of human coronavirus.

SARS-CoV-2 or COVID-19 infection has spread worldwide spanning 203 countries [8-10]. The SARS-CoV-2 infections spread rapidly between humans in short time, compelling WHO to declare it a pandemic. SARS-CoV-2 has caused more than 10,00,000 infections with $~ 55,000$ deaths and $\sim 40,000$ are in serious condition, and the numbers are increasing exponentially [www.worldometers.info/coronavirus]. Sequence alignment of human coronaviruses from various species indicates SARS-CoV exhibited the highest genome sequence identity (80\%) to SARS-CoV-2, and MERS has 50\% identity with SARS-CoV-2. SARS-CoV from bat have the highest genome sequence identity (>88\%) to SARS-CoV-2 [11]. The SARS-CoV-2 has higher infectivity than SARS-CoV because of higher binding affinity ( 20 times) of its surface $\mathrm{S}$ proteins with human angiotensin-converting enzyme II (ACE2) receptors, and efficient endocytosis into host cells after priming S1/S2 by human TMPRSS2 proteases [12, 13]. Apart from bat SARS-CoV, a recent report indicates Pangolin-CoV found in Malayan pangolins is the most closely related CoV to SARS-CoV-2 with $91 \%$ genome similarity [14].

Vaccines against SARS and MERS are not yet available. Vaccines in case of MERS were not successful as human antibodies subsided after treatment. Vaccine development against SARSCoV-2 might take time as its human response is unknown. There is an urgent need to treat SARS-CoV-2 infection. The sequences of proteins of SARS-CoV-2 share very high similarity to 
its closest coronavirus homologue, SARS-CoV, and hence many approved, pre-clinical, antiviral, anti-malarial and anti-SARS drugs are being repurposed, and even administered to patients [15-17]. Targeted therapy against the enzymes of SARS-Cov-2 with new drugs, will be a rational approach to treat the infections in long run.

SARS-CoV-2 has two proteases, papain-like protease (PLpro) and chymotrypsin like protease (3CLPro), necessary for its survival in hosts [18]. SARS-CoV-2 encodes polyprotein1 a/b (PP $\mathrm{a} / \mathrm{b}$ ) which is cleaved by these two proteases, yielding multifunctional proteins involved in transcription, replication and infection of the virions. SARS-CoV-2 PLpro cleaves first three sites at the N-terminus and 3CLpro cuts at the other 11 sites of PP a/b forming 16 non-structural proteins (Nsp) [19, 20]. Since the SARS-CoV-2 genome release the structural biology research made rapid progress, and ninety three structures of SARS-CoV-2 proteins are now available in PDB. SARS-CoV-2 PLpro crystal structure is not yet available in PDB. SARS-CoV-2 PLpro performs three proteolytic processing of the PP a/b aiding replication as well as subverts cellular ubiquitination machinery to facilitate viral survival. Hence SARS-CoV-2 PLpro is a promising drug target against SARS-CoV-2 resulting in disruption of virus replication and survival.

Our approach is targeted therapy against SARS-CoV-2 PLpro, with the goal to identify natural products as drugs. Natural products still holds a stake of more than $60 \%$ of all the FDA approved drugs, even though combinatorial synthesis coupled with molecular docking have led to many synthetic drugs [21]. Hence virtual screening of our natural product compound database was performed by docking with closed and partially open conformers of SARS-CoV-2 PLpro homology models. Here onwards partially open PLpro conformer would be denoted as open PLpro conformer. We prepared a natural product compounds library of 60 selective compounds which fit into the S3-S4 substrate binding pocket of SARS-CoV-2 PLpro. These low molecular weight compounds are found in rhizomes, Alpinia officinarum (used in Indian Ayurveda), and ginger and curcuma (used as Indian spices), and were docked. In silico results are promising as this selective class of compounds bind with high in silico affinity to SARS-CoV-2 PLpro, and are potential inhibitors against SARS-CoV-2. Our results reveal new lead compounds targeting SARS-CoV-2 PLpro, and pave way to further structure-based modifications or extract formulations of these compounds as new potent inhibitors to treat infections. Subsequent in vitro and in vivo experiments are planned to elucidate their efficacy against SARS-CoV-2. 


\section{Methods and materials}

\subsection{Amino acid sequence alignment, homology modelling and molecular docking}

Protein sequence alignment, viewing and analysis were performed using ClustalX and Jalview $[22,23]$.

The sequences of proteins of SARS-CoV-2 share very high similarity to its closest coronaviruses homologue, SARS-CoV. Homology modelling was done on Swissmodel online server using SARS PLpro crystal structures templates [24]. The 3D homology models of PLpro were build using the PDB structure templates, PDB ID 3E9S which has the ligand TTT in S3-S4 pocket $(\mathrm{GMQE}=0.95$ and QMean $=-0.22)$ and has flap closed, and PDB ID 5TL6 (GMQE $=0.92$ and QMean = -0.22) which has flap partially open.

A virtual screening of natural product compounds was performed using molecular docking with SARS-CoV-2 Plpro to find potential inhibitors. A small in-house library of natural product compounds was constructed, containing 60 compounds fitting the S3-S4 pocket of PLpro. These compounds are present in rhizomes Alpinia officinarum, and, ginger and curcuma [25-27] found in Indian sub-continent. Few compounds were synthesized in our laboratory.

Ligands were prepared using Openbabel and optimized using ACD chemsketch [28, 29]. Ligand and protein hydrogens and charges were added at $\mathrm{pH} \mathrm{7,} \mathrm{using} \mathrm{LeadIT} \mathrm{incorporated} \mathrm{in}$ BioSolveIT software suite [30]. The 60 compounds were docked into the small solvent accessible substrate binding cavity spanning S3-S4 pocket in both open and closed SARS-CoV-2 PLpro catalytic domains using LeadIT with a limit of 200 poses per compound. Top 10 docked poses per compound were extracted. Hyde calculations were carried out on each pose to obtain the binding free energy $(\Delta \mathrm{G})$ and ligand efficiency (LE) values [31]. Each docked compound was visually inspected for $\mathrm{H}$-bonds, electrostatic and hydrophobic interactions. Interaction diagrams were made using LeadIT. Structural, surface and superposition figures were made using Pymol and protein cavity sizes were determined using Mole2.0 [32, 33].

\section{Results and discussions}

\subsection{Active site and substrate binding pockets of SARS-CoV-2 PLpro homology models}

The on-going SARS-CoV-2 epidemic makes us painfully realize that our current options for treating life-threatening zoonotic coronavirus infections are very limited. Although the outbreaks of SARS in 2003 and MERS-CoV in 2012 triggered extensive research efforts, there are currently no drugs that can treat any zoonotic coronavirus. Such emerging coronaviruses will 
continue to pose a threat to global public health in the future. Vaccines and approved drugs against human coronavirus infections are absent. Therefore, finding new inhibitors targeting the essential viral proteins should be developed urgently. The sequences of proteins of SARS-CoV-2 share more than $80 \%$ similarity to SARS-CoV. Targeted therapy against SARS-CoV-2 enzymes, especially proteases will lead to direct inhibition, and more potent and specific anti-SARS-CoV2 drugs. The SARS-CoV PLpro is a potential drug target and inhibitors were designed against it targeting the substrate binding site [34]. The SARS-CoV PLpro and SARS-CoV-2 PLpro share high sequence homology as shown in figure 1.

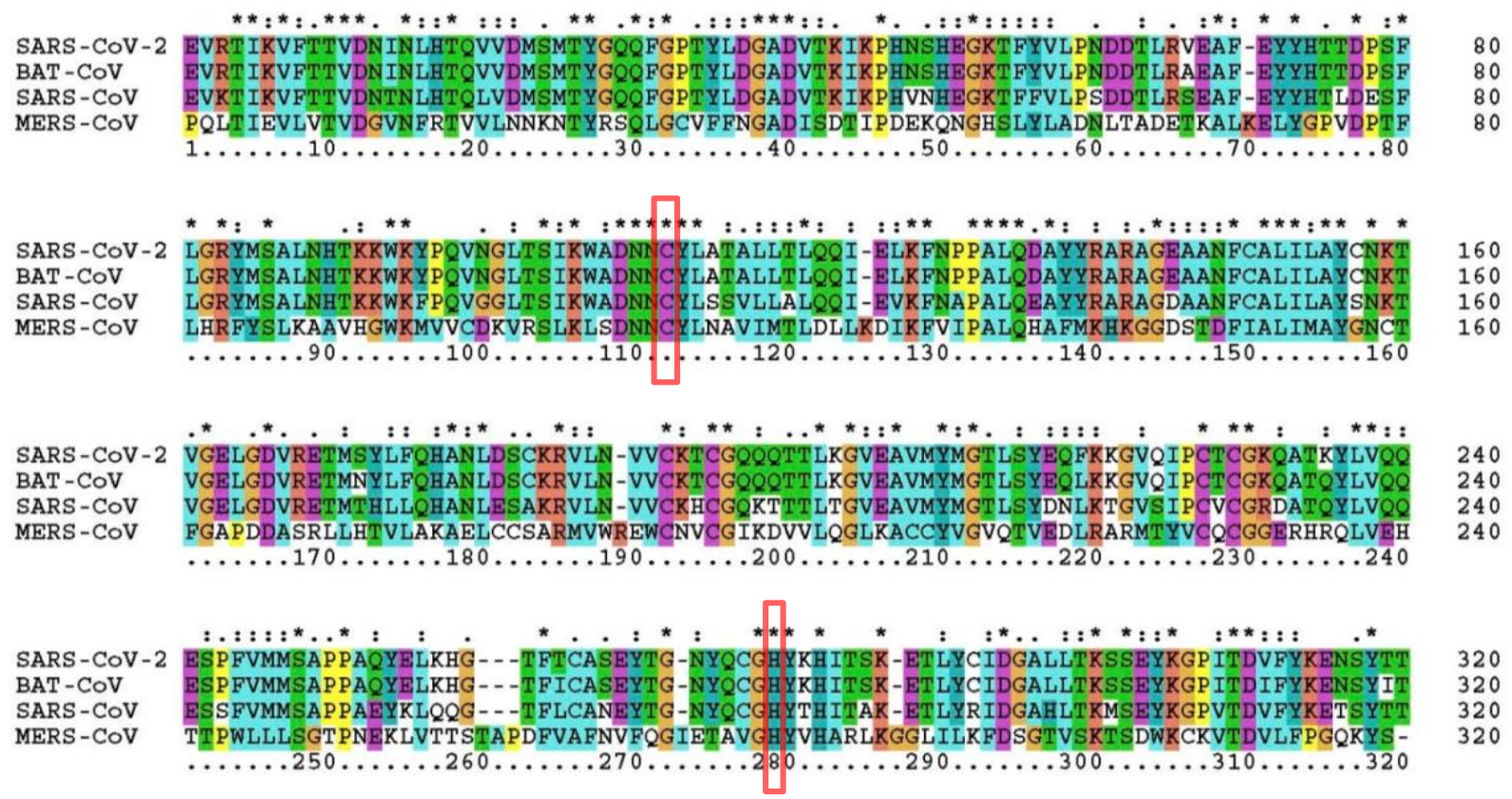

Figure 1: Superposition of the amino acid sequences of PLpro of SARS-CoV-2 with bat, SARS and MERS CoVs. Catalytic dyads, C114 and $\mathrm{H} 275$ are enclosed in red boxes.

The closed and open PLpro models superpose well with backbone $0.5 \AA$ rmsd as seen in figure 2 . Close to the docking site, the enzyme active site is located as shown in figure 2. The PLpro active site is similar to cysteine proteases where the catalytic dyad residues $\mathrm{C} 114$ and $\mathrm{H} 275$ are located spanning the S1/S1' pocket (figures 2 and 3). The important residues involved in catalysis are shown in figure 3. 


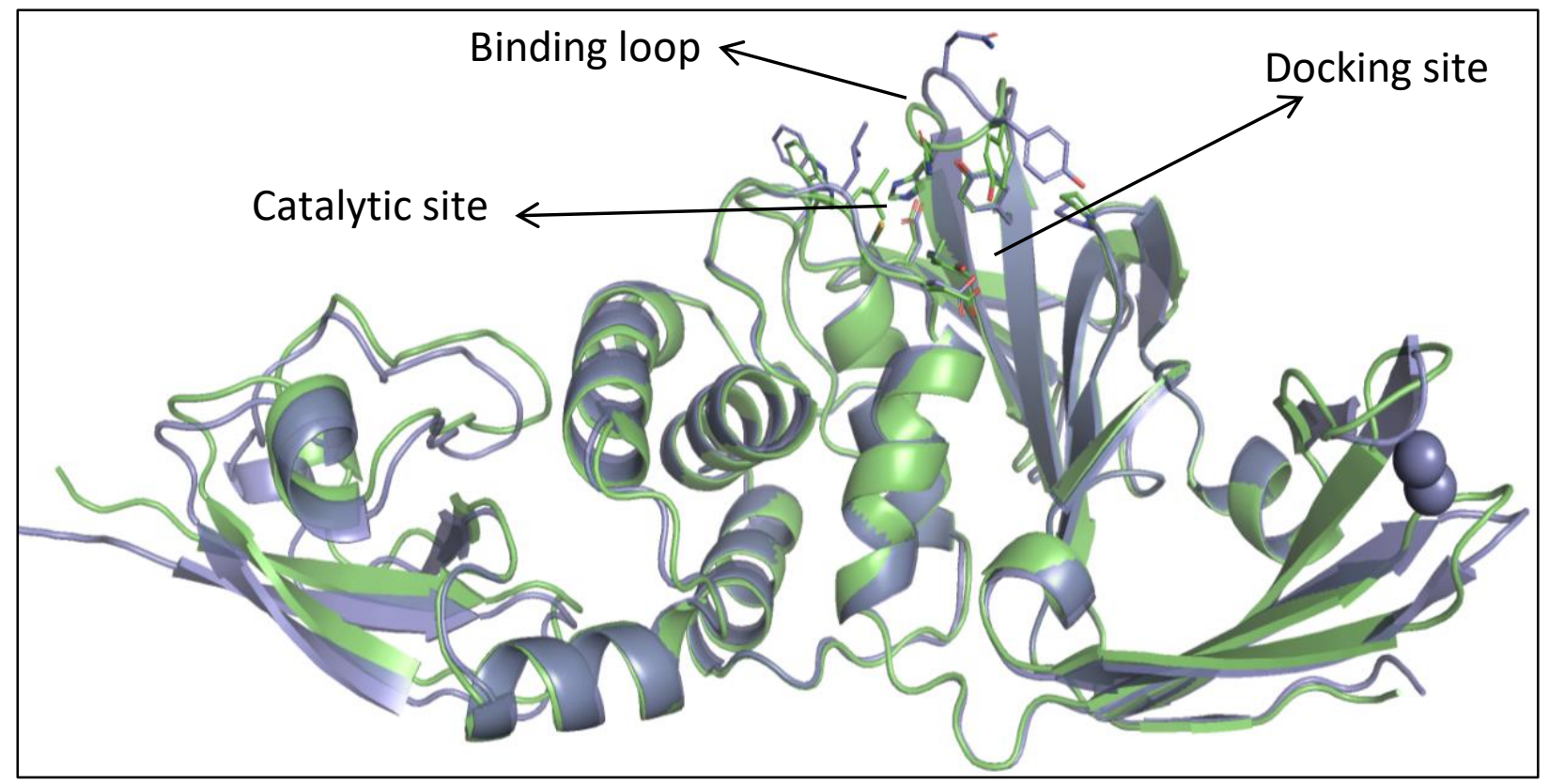

Figure 2. Superposition of the closed (green) and partially open (blue) SARS-CoV-2 PLpro models used in docking. The protease residues in active and docking sites are shown in sticks.

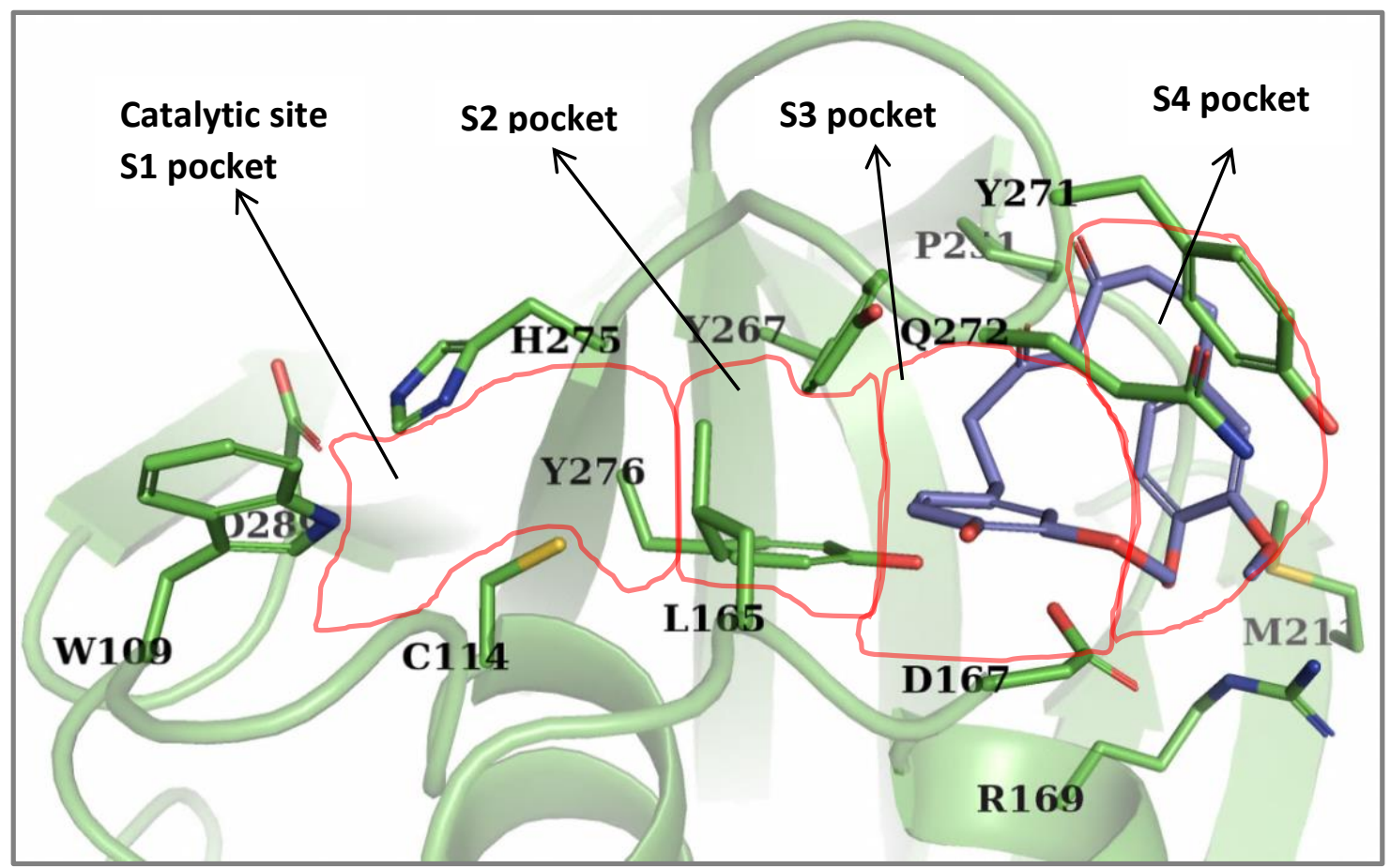

Figure 3. Cartoon of SARS-CoV-2 PLpro protein backbone showing the active site and docking site residues in green sticks. Substrate binding pockets are shown in freehand red enclosures. One docked compound (blue sticks) is shown in the S3-S4 pocket. 
The H275 deprotonates catalytic C114 when substrate peptide binds, and the generated thioanionic hole is stabilized by the nearby tryptophan, W109 residue. Nucleophilic attack by C114 thioanion on scissile peptide carbonyl, generates a covalent intermolecular tetrahedral intermediate [20]. The aspartic acid, D289 might be involved in the covalent bond cleavage enabling N-terminal peptide to exit. Substrate binding pockets are shown as S1-S4 in figure 3 within red enclosures. The region near C114 spanning S1-S1' pocket is narrow and is not solvent exposed, hence drugs cannot not bind to that pocket in closed PLpro conformer. It was shown that highly analogous SARS PLpro can accommodate only di-gly containing peptides at P1-P1', and recognizes the consensus cleavage sequence $\mathrm{LXGG}$, which is also the consensus sequence recognized by cellular de-ubiquitinating enzymes [35]. S2 pocket is only partially solvent exposed. The binding pocket S3-S4 is small, spanning from G166 to M211 and P251, where the inhibitors can dock (figure 3). S3-S4 pockets 1 in both conformers have the largest volumes as shown in figure 4 (closed PLpro volume $=851 \AA^{3}$ and open PLpro volume $=843 \AA^{3}$ ), and volumes are similar due to curling of flap in open form is away from pocket 1 . The volume of next largest pocket 2 in PLpro open conformer is $111 \AA^{3}$ more than that of closed conformer. The pocket 2 contains the catalytic residues, and are not solvent accessible in both closed and open models, which can be seen in surface representations in figure 5. The flexible substrate binding loop containing Q272 and Y271 and connecting $\beta$-sheets are expected to open up more to accommodate the substrate PP a/b chain for cleavage (figures 2 and 5). Hence our open model is possibly partially open model.
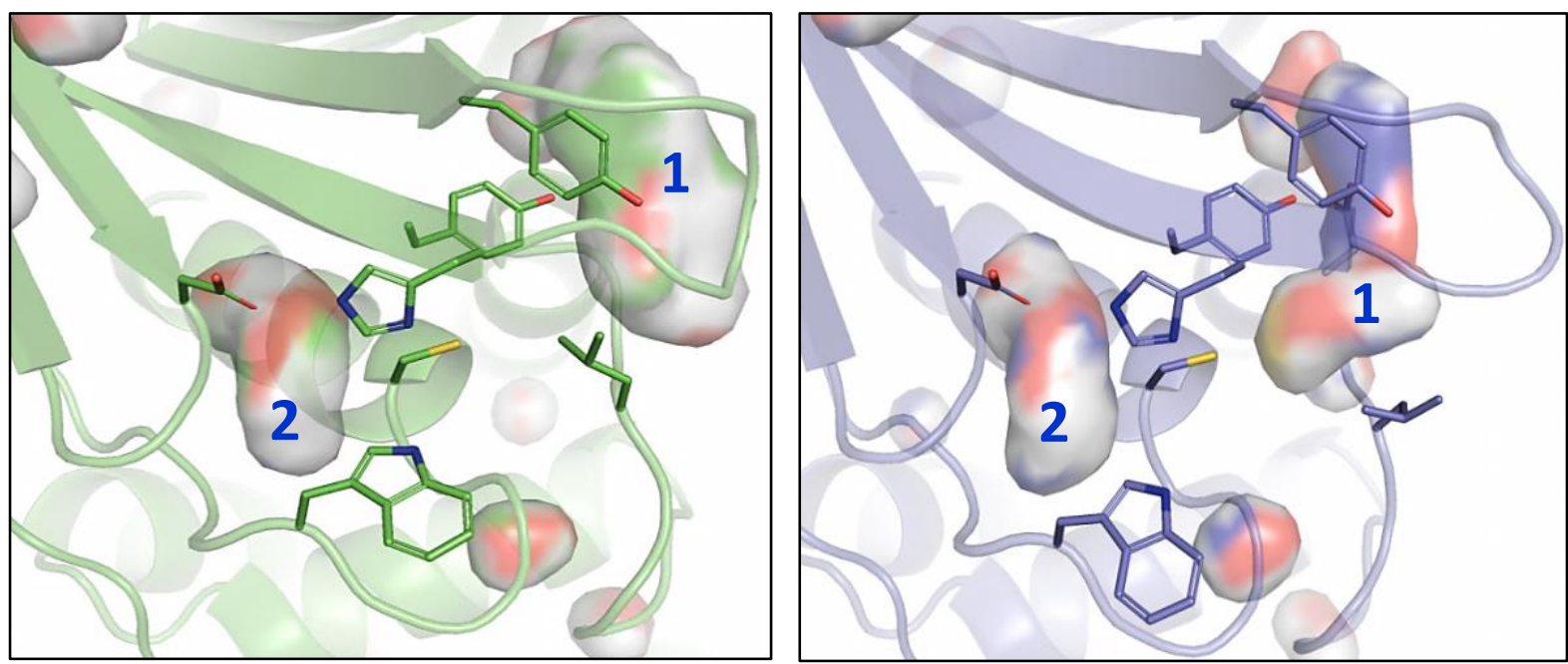

Figure 4. Cavities in the closed (left, volume $=851$ (docking site) / 338 (catalytic site) $\AA^{3}$ ) and partially open (right, volume $=843$ (docking site) $/ 449$ (catalytic site) $\AA^{3}$ ) SARS-CoV-2 PLpro homology models. Ligands were docked in the larger cavity 1. 

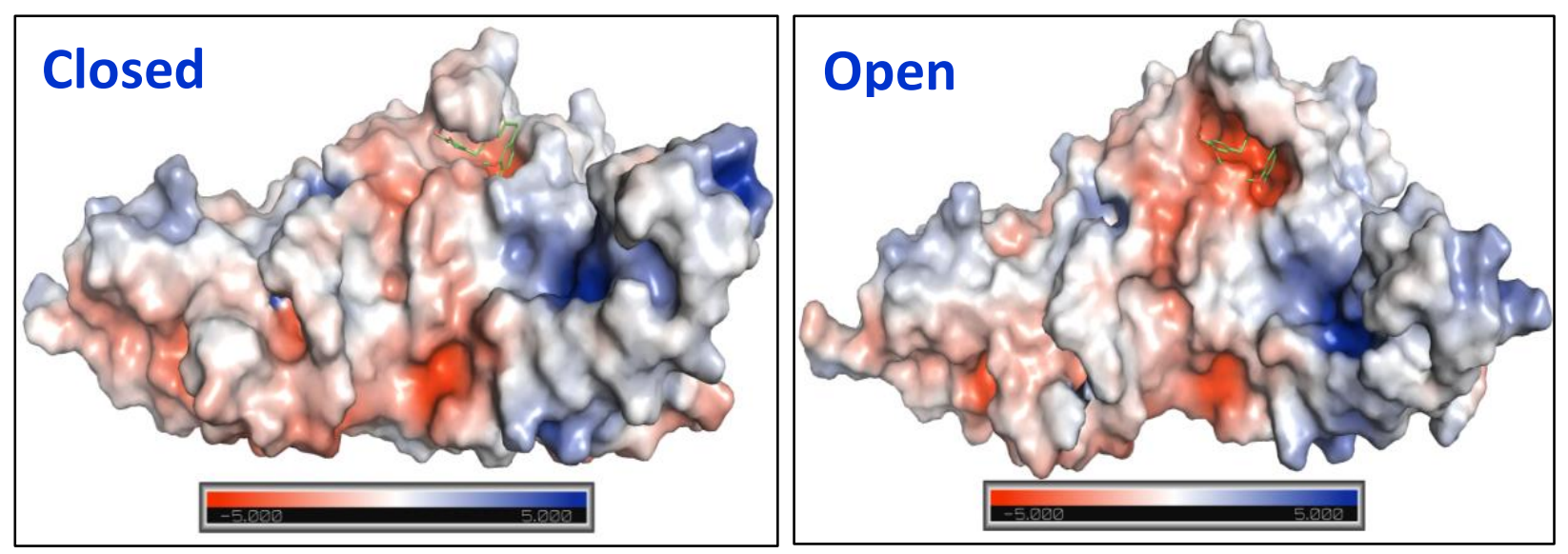

Figure 5. Charged surface of the closed (left) and open (right) conformer of PLpro models. A docked ligand spanning the S3-S4 pocket is shown in green sticks. It is seen that the catalytic site pocket 2 is not exposed to solvent.

We docked our ligands in S3-S4 pocket of both closed and open conformers. The 270s substrate binding loop, locked in an open conformation by inhibitors would also inhibit protease action, as seen in HIV-1 protease [36].

\subsection{Natural product compound docking against SARS-CoV-2 PLpro}

Two homology models of SARS-CoV-2 PLpro were used in molecular docking, one having closed and other partially open conformations. To search for potential therapeutic drugs against SARS-CoV-2 PLpro, we virtually screened a library of natural product compounds. The 60 compounds used in docking are active components of rhizomes from Indian sub-continent, Alpinia officinarum and, ginger and curcuma used as Indian spices [25-27]. Currently HIV-1 protease drugs, lopinavir and ritonavir are being administered to patients infected with SARSCoV-2. To compare the docking results with our natural product compound library, we docked all US FDA approved HIV-1 protease inhibitors and all PLpro ligands available in PDB with closed and open conformers of PLpro. Lopinavir and ritonavir did not give good docking scores. We found that only one ligand, TTT in PDB ID 3ZYE binds with high affinity $(\Delta \mathrm{G}=-50 \mathrm{~kJ} / \mathrm{mol}$ and LE $=0.49$ ) to closed PLpro model, but shows poor binding to the open model. We find that the binding affinities of other drugs were less that $-24 \mathrm{~kJ} / \mathrm{mol}$ and LE less than 0.20.

Table 1 shows the $\Delta \mathrm{G}$ and $\mathrm{LE}$ values of the docked compounds having $\Delta \mathrm{G} \leq-30 \mathrm{~kJ} / \mathrm{mol}$ with closed PLpro conformer. Corresponding values for open conformer are shown in the line below. 
Table 1. List of natural product compounds docked against SARS-CoV-2 PLpro with $\Delta G$ and $L E$ values. Open indicates the values with open conformer. Ligands are sorted from highest to lowest affinity with closed PLpro, where compounds 1-14 belong to Alpinia officinarum, 15-17 belong to ginger and 18-19 belong to curcuma rhizomes.

No Compound
$\Delta \mathbf{G}$

$(\mathbf{k J} / \mathbf{m o l})$
Binding LE $\quad \log P$

affinity
116

Open<smiles>COc1cc(CC/C=C/C(=O)CCc2ccccc2)ccc1O</smiles>

213

Open<smiles>COc1cc(CCCC(=O)CCc2ccccc2)ccc1O</smiles>

345

Open<smiles>COc1cc(CCC(=O)CCC(=O)CCc2ccc(O)c(OC)c2)ccc1O</smiles>

$4 \quad 36$

Open<smiles>CC(=O)OC(CCCCCc1ccc(O)cc1)CCc1ccc(O)c(O)c1</smiles>

$5 \quad 22$

Open<smiles>OC(CCC(O)c1ccccc1)CC(O)c1ccccc1</smiles>

$6 \quad 34$

Open<smiles></smiles>

$\begin{array}{ll}7 & 10\end{array}$

Open<smiles>COC(CCc1ccc(O)cc1)CC(=O)CCc1ccccc1</smiles>

843
$-47$

$-28$

$-45$

$-28$

$-45$

$-44$

$-28$

$-43$

$-27$

$-40$

$-22$

$-39$

$-29$

$-39$

$-29$
$1 \mathrm{nM}$

0.49

$3.80 \pm 0.28$

$10-100 \mu \mathrm{M}$

0.27

$1 \mathrm{nM}$

0.45

$2.52 \pm 0.35$

$10-100 \mu \mathrm{M} \quad 0.26$

$1 \mathrm{nM}$

0.43

$2.13 \pm 0.13$

No binding

$$
\text { 1-10 nM }
$$

0.42

$4.13 \pm 0.49$

$$
10-100 \mu \mathrm{M} \quad 0.26
$$

$$
\text { 1-10 nM }
$$

0.45

$1.10 \pm 0.74$

$10-100 \mu \mathrm{M} \quad 0.28$

$1-10 \mathrm{nM}$

0.36

$0.77 \pm 0.14$

$>100 \mu \mathrm{M}$

0.20

10-100 nM

0.41

$3.64 \pm 0.27$

$10-100 \mu \mathrm{M} \quad 0.30$<smiles>COc1cc(CCC(=O)CC(=O)CC(O)c2ccc(O)cc2)ccc1O</smiles>

10-100 nM

0.36

$1.74 \pm 0.47$ 
(kJ/mol) affinity

\section{Open}

941

Open<smiles>O=C(CCc1ccc(O)cc1)CC(=O)CC(O)c1ccc(O)cc1</smiles>

$10 \quad 44$

Open<smiles>COc1cc(CCC(=O)CC(=O)CC(O)c2ccc(O)c(O)c2)ccc1O</smiles>

$11 \quad 51$

Open<smiles>COc1cc(CCC(O)CC(=O)CCc2ccccc2)ccc1O</smiles>

$12 \quad 48$

Open<smiles>O=C(CCC(=O)CCc1ccc(O)c(O)c1)CCc1ccc(O)cc1</smiles>

138

Open<smiles>COc1cc(CCC(O)CC(=O)CCc2ccc(O)cc2)ccc1O</smiles>

$14 \quad 42$

Open<smiles>COc1cc(CCC(=O)CC(=O)CCc2ccc(O)cc2)ccc1O</smiles>

15 8Gingerol

Open

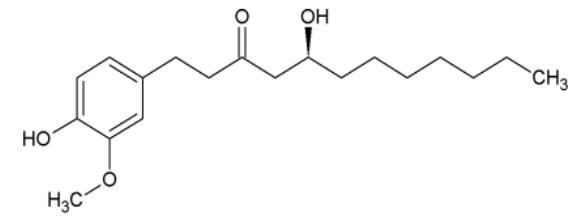

16 10Gingerol

Open
$-38$

$-28$

$-38$

$-31$

$-35$

$1 \mu \mathrm{M}-100 \mathrm{nM} \quad 0.35$

$2.52 \pm 0.35$

no binding

$\begin{array}{llll}-34 & 1 \mu \mathrm{M}-100 \mathrm{nM} & 0.34 & 2.11 \pm 0.41\end{array}$

$-35 \quad 1 \mu \mathrm{M}-100 \mathrm{nM} \quad 0.35$

$-3$

$-20$

$$
1 \mu \mathrm{M}-100 \mathrm{nM} \quad 0.32
$$

$1.79 \pm 0.35$

$>100 \mathrm{uM} \quad 0.20$
$-30$

$-24$

$3.55 \pm 0.27$

$-15>100 \mu \mathrm{M} \quad 0.16$

$-42$

$1-10 \mathrm{nM} \quad 0.40$

$3.85 \pm 0.27$

$-21 \quad>100 \mu \mathrm{M} \quad 0.21$

$2.42 \pm 0.42$

0.22

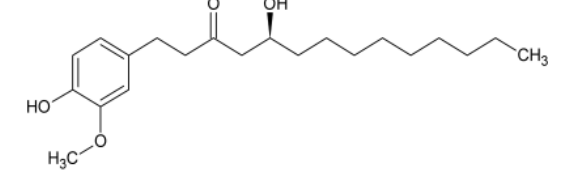


17 6Gingerol

Open

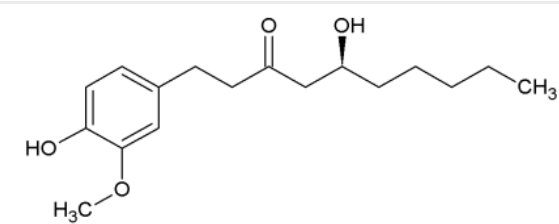

18 Curcumin Open

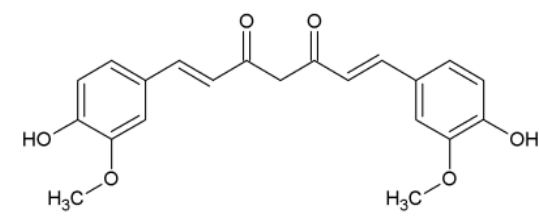

19 Cur2 Open

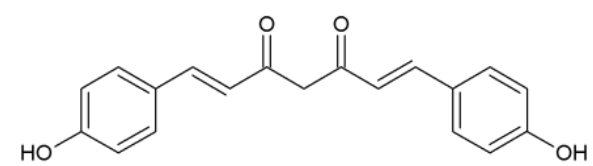

20 Chloroquine Open

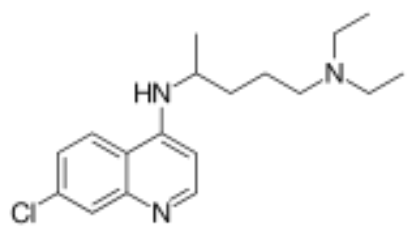

21 Hydroxychloroquine Open

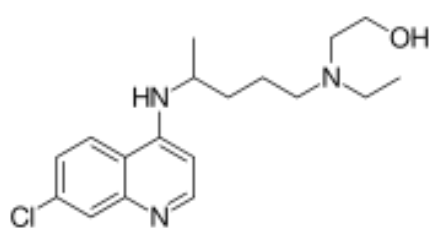

$-39$

$-26$

$$
\text { 1-10 nM }
$$

0.40

$2.49 \pm 0.27$

$10-100 \mu \mathrm{M} \quad 0.30$

$-34$

$1 \mu \mathrm{M}-100 \mathrm{nM} \quad 0.30$

$2.92 \pm 0.48$

$-23$

$$
>100 \mu \mathrm{M}
$$

0.20

$-31$

10-1 $\mu \mathrm{M}$

0.32

$3.39 \pm 0.44$

$-24$

$10-100 \mu \mathrm{M} \quad 0.25$

$-27$

$100 \mu \mathrm{M}-10 \mu \mathrm{M} \quad 0.29$

4.63

$-26$

$100 \mu \mathrm{M}-10 \mu \mathrm{M} \quad 0.28$

$-36$

$1 \mu \mathrm{M}-100 \mathrm{nM} \quad 0.37$

2.89

$-32 \quad 1 \mu \mathrm{M}-100 \mathrm{nM} \quad 0.33$

We also docked the currently administered anti-malarial drugs chloroquine and hydroxychloroquine, shown as compounds nos. 20 and 21 in table 1 [37]. Hydroxy-chloroquine binds with moderate affinity to both closed and open conformer of PLpro as compared to Chloroquine. Overall we find that most of the compounds bind with lowest $\Delta \mathrm{G}$ values to closed conformer of PLpro. The compounds also bind to open conformer but with higher $\Delta \mathrm{G}$ values. Interestingly few compounds are docked partly in the S2 pocket in the open PLpro conformer. Our docking results will be based on closed Plpro conformer. In table 1, we list the 14 compounds (1-14) which are active components of Alpinia officinarum, 15-17 belong to ginger, and 18-19 belong to curcuma rhizomes, which bind with high affinity $(\Delta \mathrm{G}>-30 \mathrm{~kJ} / \mathrm{mol})$ to closed PLpro.

We predict compounds 16, 13, 45, 36 and 22 from Alpinia officinarum, and 8-Gingerol, 10Gingerol and 6-Gingerol from ginger are potent inhibitors of PLpro, having nM binding affinity 
and high LE values. Curcumin and its derivative Cur2 bind to PLpro better than the anti-malarial drugs, but have lower LE values than the above eight compounds. The eight docked molecules on protease surface and their interactions with protease residues are shown in figure 6. The docking site surfaces in the open and closed PLpro are negatively charged (figure 5). The docked compounds make 3-5 hydrogen bonds and have favourable electrostatic and hydrophobic interactions with PLpro residues (figure 6) contributing to their low $\Delta \mathrm{G}$ values.
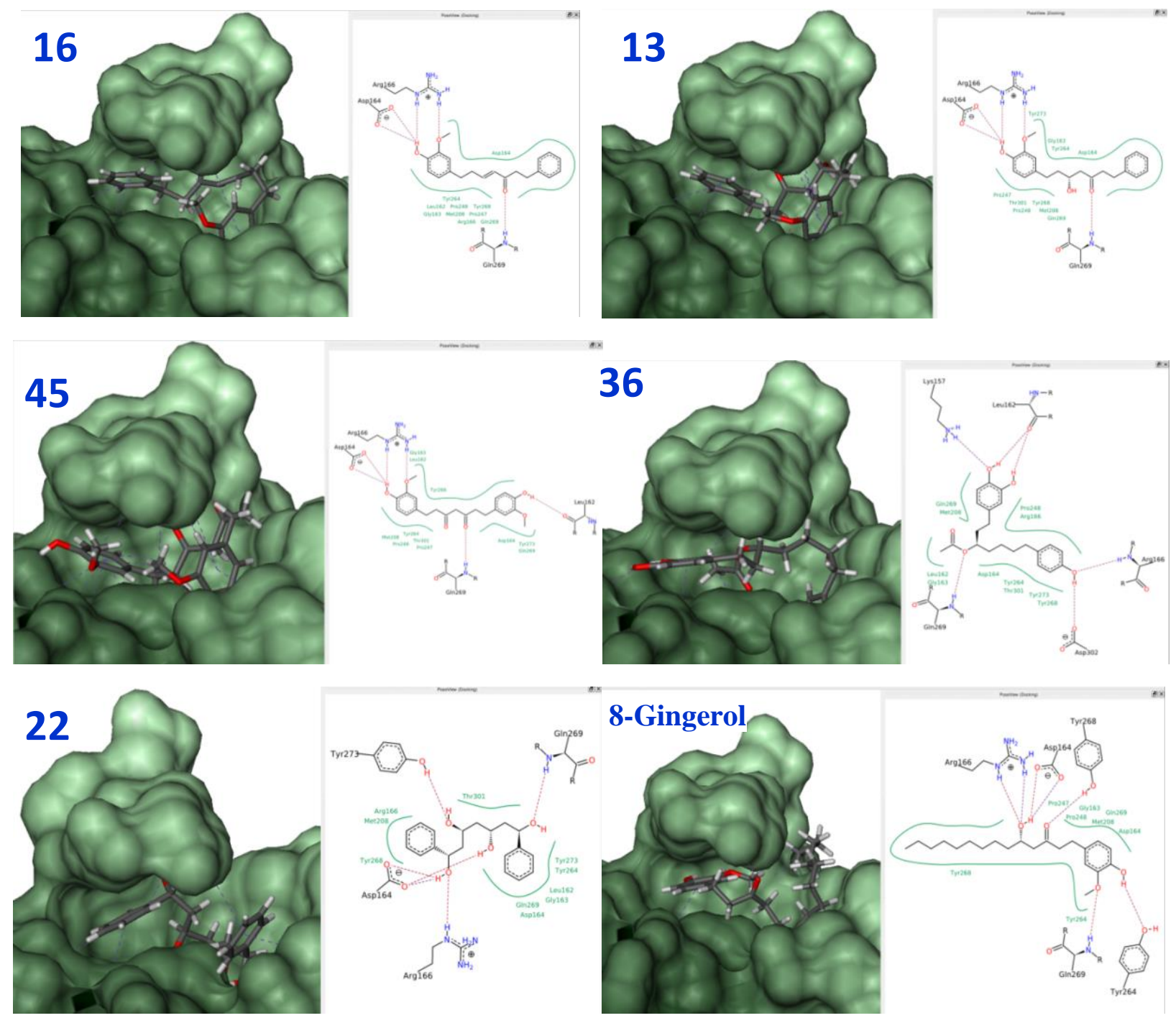

10-Gingerol 


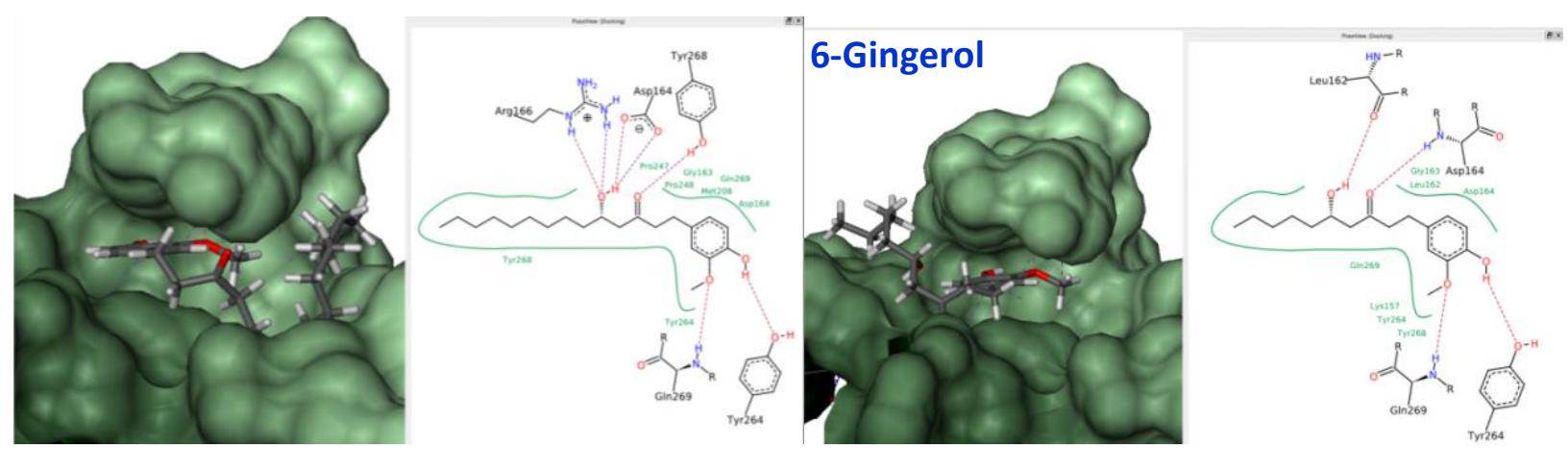

Figure 6. The eight docked compounds (name in inset) are potential inhibitors of SARS-CoV-2 Plpro. They are bound to the closed conformer surface (left) and their interactions are shown with PLpro residues (right).

Our in silico results indicate the selective class of compounds found in rhizomes of Alpinia officinarum and ginger, bind with high affinity (nM range) to SARS-Cov-2 PLpro.These compounds are one of the major component in the rhizomes. In India these rhizomes are extensively used in cooking, to treat infections and taken during cough and cold. This correlates with the fact that SARS-CoV-2 infections are less in India, and recovery is expected to be more. Therefore, natural product compounds or rhizome extract concentrates containing these compounds, can be used to treat of SARS-CoV-2 infections. Natural product compounds from plants will be a good start point since they are already being consumed. Also they provide a skeletal know how to the synthetic chemists to further modify them as more potent anti-SARSCoV-2 drugs. Few of these compounds are synthesized in our laboratory, and further in vivo and in vitro anti-viral testing are planned. Our results are shared with the scientific community to urgently develop formulations and anti-SARS-CoV-2 inhibitors and provide immediate relief to infected patients.

\section{Conclusions}

The in house library of natural product compounds found in rhizome family, Alpinia officinarum, ginger and curcuma, were docked against the closed and partially open conformers of SARSCoV-2 PLpro homology models. Eight compounds found in rhizomes of Alpinia officinarum and ginger were identified as potential inhibitors of SARS-CoV-2 PLpro. Our study on structure based molecular docking show these natural product inhibitors are promising drug candidates and potential use of their rhizome extracts against SARS-CoV-2.

\section{Acknowledgments}


Our research is supported by National Facility for Structural Biology, BARC and Department of Atomic Energy.

\section{References}

1. Cheng VC, Lau SK, Woo PC, Yuen KY. Severe acute respiratory syndrome coronavirus as an agent of emerging and reemerging infection. Clin. Microbiol. Rev. (2007) 20: 660-94.

2. Lee N, Hui D, Wu A, Chan P, Cameron P, Joynt GM, et. al. A major outbreak of severe acute respiratory syndrome in Hong Kong. N. Engl. J. Med. (2003) 348: 1986-94.

3. Zaki AM, van Boheemen S, Bestebroer TM, Osterhaus AD, Fouchier RA. Isolation of a novel coronavirus from a man with pneumonia in Saudi Arabia. N. Engl. J. Med. (2012) 367: 1814-20.

4. Lau SK, Woo PC, Li KS, Huang Y, Tsoi HW, Wong BH, et. al. Severe acute respiratory syndrome coronavirus-like virus in Chinese horseshoe bats. Proc. Natl. Acad. Sci. USA (2005) 102: $14040-5$.

5. Reusken CB, Haagmans BL, Müller MA, Gutierrez C, Godeke GJ, Meyer B, et.al. Middle East respiratory syndrome coronavirus neutralising serum antibodies in dromedary camels: a comparative serological study. Lancet Infect. Dis. (2013) 13: 859-66.

6. Li W, Moore MJ, Vasilieva N, Sui J, Wong SK, Berne MA, et. al. Angiotensin-converting enzyme 2 is a functional receptor for the SARS coronavirus. Nature (2003) 426: 450-4.

7. Bosch BJ, van der Zee R, de Haan CA, Rottier PJ. The coronavirus spike protein is a class I virus fusion protein: structural and functional characterization of the fusion core complex. $J$. Virol. (2003) 77: 8801-11.

8. Zhu N, Zhang D, Wang W, Li X, Yang B, Song J, et. al. A novel coronavirus from patients with pneumonia in China, 2019. N. Engl. J. Med. (2020) 382: 727-733.

9. Chen Y, Liu Q, Guo D. Emerging coronaviruses: genome structure, replication, and pathogenesis. J. Med. Virol. (2020) 92(4):418-423.

10. Huang C, Wang Y, Li X, Ren L, Zhao J, Hu Y, et al. Clinical features of patients infected with 2019 novel coronavirus in Wuhan, China. The Lancet (2020) 395: 497-506.

11. Zhou P, Yang X, Wang X, et. al. A pneumonia outbreak associated with a new coronavirus of probable bat origin. Nature (2020) 579: 270-273.

12. Wrapp D, Wang N, Corbett KS, Goldsmith JA, Hsieh CL, Abiona O, Graham BS, McLellan JS, D. Wrapp. Cryo-EM structure of the 2019-nCoV spike in the prefusion conformation. Science (2020) 367(6483):1260-1263.

13. Ou X, Liu Y, Lei X, et. al. Characterization of spike glycoprotein of SARS-CoV-2 on virus entry and its immune cross-reactivity with SARS-CoV. Nat. Commun. (2020) 11: 1620. DOI: 10.1038/s41467-020-15562-9. 
14. Zhang T, Qunfu W, Zhang Z. Probable pangolin origin of SARS-CoV-2 associated with the COVID-19 outbreak. Current Biology (2020). DOI: 10.1016/j.cub.2020.03.022.

15. Zhou Y, Hou Y, Shen J, et. al. Network-based drug repurposing for novel coronavirus 2019nCoV/SARS-CoV-2. Cell Discov. (2020) 6: 14. DOI: 10.1038/s41421-020-0153-3.

16. Arya R, Das A, Prashar V, Kumar M. Potential inhibitors against papain-like protease of novel coronavirus (SARS-CoV-2) from FDA approved drugs. ChemRxiv (2020), chemrxiv.org/articles/11860011.

17. Kupferschmidt K, Cohen J. Race to find COVID-19 treatments accelerates. Science (2020) 27 (367): 1412-1413.

18. Guangdi L, Clercq ED. Therapeutic options for the 2019 novel coronavirus (2019-nCoV). Nature Rev. Drug Discov. (2020) 19: 149-150.

19. Kiemer L, Lund O, Brunak S, Blomcorresponding N. Coronavirus 3CLproproteinase cleavage sites: Possible relevance to SARS virus pathology. BMC Bioinformatics (2004) 5: 72.

20. Ratia K, Singh SK, Bernard D, Santarsiero NB, Baker SC, Stevens RC, Mesecar AD. Severe acute respiratory syndrome coronavirus papain-like protease: Structure of a viral deubiquitinating enzyme. Proc. Natl. Acad. Sci. USA (2006) 103 (15): 5717-5722.

21. Cragg GM., Grothaus PG, Newman DJ. Impact of Natural Products on Developing New Anti-Cancer Agents. Chem. Rev. (2009) 109: 3012-3043.

22. Larkin MA, Blackshields G, Brown NP, Chenna R, McGettigan PA, McWilliam H, Valentin F, Wallace IM, Wilm A, Lopez R, Thompson JD, Gibson TJ, Higgins DG. Clustal W and Clustal $X$ version 2.0. Bioinformatics (2007) 23: 2947-2948.

23. Waterhouse AM, Procter JB, Martin DMA, Clamp M, Barton GJ Jalview Version 2-a multiple sequence alignment editor and analysis workbench. Bioinformatics (2009) 25: 11891191.

24. Waterhouse A, Bertoni M, Bienert S, Studer G, Tauriello G, Gumienny R, Heer FT, de Beer TAP, Rempfer C, Bordoli L, Lepore R, Schwede T. SWISS-MODEL: homology modelling of protein structures and complexes. Nucleic Acids Res. (2018) 46(W1): W296-W303.

25. Matsuda H, Ando S, Kato T, Morikawa T, Yoshikawa M. Inhibitors from the rhizomes of Alpinia officinarum on production of nitric oxide in lipopolysaccharide-activated macrophages and the structural requirements of diarylheptanoids for the activity. Bioorg. Med. Chem. (2006) 14: $138-142$.

26. Rüedi P, Juch M. Chemistry and biological activities of long-chain alkyloxy-catechols of the [n]-gingerol-type. Curr. Org. Chem. (1999) 3: 623-646.

27. Ruby A J, Kuttan G, Balu KVD, Rajasekharan KN, Kuttan R. Anti-tumour and antioxidant activity of natural curcuminoids. Cancer Lett. (1995) 94: 79-83. 
28. O'Boyle NM, Banck M, James CA, Morley C, Vandermeersch T, and Hutchison GR. "Open Babel: An open chemical toolbox. J. Cheminform. (2011), 3: 33.

29. ACD/Labs 2015, C10E41, Advanced Chemistry Development, Inc., Toronto, On, Canada, www.acdlabs.com.

30. LeadIT version 2.3.2; BioSolvelT GmbH, Sankt Augustin, Germany, 2019, www.biosolveit.de/LeadIT

31. Hyde: Lange G, Klein R, Albrecht J, Rarey M, Reulecke I (2010), Patent Nr. EP2084520

32. The PyMOL Molecular Graphics System, Version 2.0 Schrödinger, LLC.

33. Sehnal D, Svobodová VR, et. al. MOLE 2.0: advanced approach for analysis of biomacromolecular channels. J Cheminform. (2013) 5: 39.

34. Ghosh AK, Takayama J, Rao KV, Ratia K, Chaudhuri R, Mulhearn DC, Hyun L, et. al. Severe acute respiratory syndrome coronavirus papain-like novel protease inhibitors: design, synthesis, protein-ligand X-ray structure and biological evaluation. J. Med. Chem. (2010) 53: 4968-4979.

35. Barretto N, Jukneliene D, Ratia K, Chen Z, Mesecar AD, Baker SC. The papain-like protease of severe acute respiratory syndrome coronavirus has deubiquitinating activity. J. Virol. (2005) 79(24): 15189-15198.

36. Bçttcher J, Blum A, Dçrr S, Heine A, Diederich WE, Klebe G. Targeting the Open-Flap Conformation of HIV-1 Protease with Pyrrolidine-Based Inhibitors. Chem. Med. Chem. (2008) 3: $1337-1344$.

37. Liu J, Cao R, Xu M. et. al. Hydroxychloroquine, a less toxic derivative of chloroquine, is effective in inhibiting SARS-CoV-2 infection in vitro. Cell Discov. (2020) 6: 16. DOI: 10.1038/s41421-020-0156-0. 